\title{
Bifacial Silicon Solar Cell Steady Photoconductivity under Constant Magnetic Field and Junction Recombination Velocity Effects
}

\author{
Amadou Diao', Mamadou Wade², Moustapha Thiame³, Grégoire Sissoko \\ ${ }^{1}$ Semiconductors and Solar Energy Laboratory, Department of Physics, Faculty of Sciences and Techniques, University of Cheikh \\ Anta Diop, Dakar, Senegal \\ ${ }^{2}$ Laboratory of Sciences and Techniques of Water and Environment, Polytechnic School of Thiès, Thiès, Senegal \\ ${ }^{3}$ University Assane SECK, Ziguinchor, Senegal \\ Email: gsissoko@yahoo.com
}

How to cite this paper: Diao, A., Wade, M., Thiame, M. and Sissoko, G. (2017) Bifacial Silicon Solar Cell Steady Photoconductivity under Constant Magnetic Field and Junction Recombination Velocity Effects. Journal of Modern Physics, 8, 2200-2208. https://doi.org/10.4236/jmp.2017.814135

Received: November 9, 2017

Accepted: December 17, 2017

Published: December 20, 2017

Copyright () 2017 by authors and Scientific Research Publishing Inc. This work is licensed under the Creative Commons Attribution International License (CC BY 4.0). http://creativecommons.org/licenses/by/4.0/

\begin{abstract}
The paper reported a theoretical study on the photoconductivity of a bifacial silicon solar cell under polychromatic illumination and a constant magnetic field effect. By use of the continuity equation in the base of the solar cell maintained in a constant temperature at $300 \mathrm{~K}$, an expression of the excess minority carriers' density was determined according to the applied magnetic field, the base depth and the junction recombination velocity. From the expression of the minority carriers' density, the photoconductivity of the solar cell was deduced and which allowed us to predict some recombination phenomena, the use of such solar cell in optoelectronics. The profile of the photoconductivity also permitted us to utilize a linear model in order to determine an electrical capacitance that varied with magnetic field.
\end{abstract}

\section{Keywords}

Bifacial Solar Cell, Photoconductivity, Junction Recombination Velocity, Magnetic Field, Electrical Capacitance

\section{Introduction}

The photoconductivity is one of the important characteristic quantities of solar cells or semiconductors [1] [2] [3] [4]. It occurs when a change of electrical conductivity is noted in an illuminated semiconductor; it also provides information on the existence of recombination centers and the distribution of the deep 
levels states in the forbidden band. So, in transient regime [5] [6], there is a relationship between the photoconductivity, the lifetime and the generation rate of the photo-generated minority carriers, that leads to the density of states determination and the existence of two types of recombination centers: monomolecular and bimolecular. While in steady-state [7]-[13], in one hand, without an applied magnetic field, the effective lifetime of minority carriers is determined and so the photoconductivity according to the temperature, the generation rate and the incident photon flux (light intensity). This has led to the determination of the density of states. On the other hand, with an applied magnetic field, the photoconductivity [14] [15] is obtained according to the wavelength, the magnetic field and the illumination power. In this work, a theoretical calculation is carried out, by use of the Drüde model and the continuity equation of the minority carriers to establish a relationship between the photoconductivity, the applied magnetic field, the base depth and the junction recombination velocity of a silicon solar cell.

\section{Theory}

We consider an $\mathrm{n}^{+}-\mathrm{p}-\mathrm{p}^{+}$type of a bifacial silicon solar cell [16] [17], in 3D, in Figure 1, where $d$ and $H$ are respectively the emitter thickness and the solar cell base thickness where a constant magnetic field $\boldsymbol{B}=$ Cte [18] [19] [20] is applied following the $y$-axis.

By illuminating the solar cell, through the emitter considered as transparent to incident light because of its very small thickness $(1 \mu \mathrm{m})$, generation and recombination of electron-hole pairs occur in the base. The excess photo-generated minority carriers (electrons) in the base, are subjected to the electrical force $q \boldsymbol{E}$, magnetic force $q \boldsymbol{v} \wedge \boldsymbol{B}$ and frictional force $-\frac{m}{\tau} \boldsymbol{v}$ during their diffusion in the crystal lattice. By use of the basic low, one obtains Equation (1):

$$
m \frac{\mathrm{d} \boldsymbol{v}}{\mathrm{d} t}=q \boldsymbol{E}+q \boldsymbol{v} \wedge \boldsymbol{B}-\frac{m}{\tau} \boldsymbol{v}
$$

where $q$ and $\tau$ are respectively the elementary charge and the average lifetime of electrons in the base; $v$ is the electron velocity; $\boldsymbol{E}$ the electric field resulting

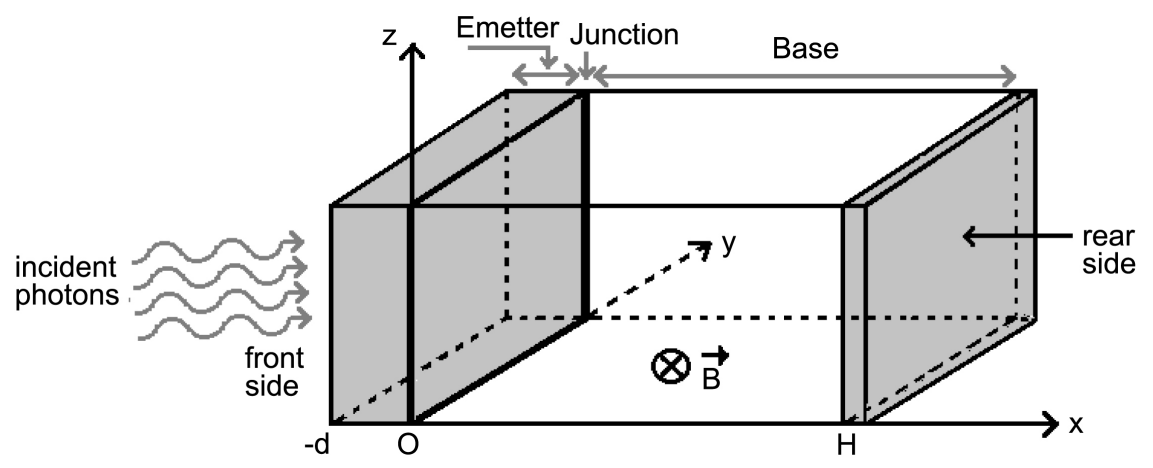

Figure 1. A schematic structure of a bifacial silicon solar cell under an magnetic field. 
from the base polarization.

In quasi-steady-state, the time derivative of $\boldsymbol{v}$ vanishes [21] [22]. Then, the Equation (1) becomes:

$$
q \boldsymbol{E}+q \boldsymbol{v} \wedge \boldsymbol{B}-\frac{m}{\tau} \boldsymbol{v}=\mathbf{0}
$$

Let's pose:

$$
\begin{gathered}
\boldsymbol{E}=E_{x} \boldsymbol{i}+E_{y} \boldsymbol{j}+E_{z} \boldsymbol{k} \\
\boldsymbol{v}=v_{x} \boldsymbol{i}+v_{y} \boldsymbol{j}+v_{z} \boldsymbol{k} \text { and } \boldsymbol{B}=B \boldsymbol{j}
\end{gathered}
$$

Substituting Equations (3) and (4) in Equation (2), and rearranging, we obtain the velocity components:

$$
\begin{gathered}
v_{x}=\frac{\mu_{o}}{\left(1+\mu_{o}^{2} B^{2}\right)} E_{x}-\frac{\mu_{o}^{2} B}{\left(1+\mu_{o}^{2} B^{2}\right)} E_{z} \\
v_{y}=\mu_{o} E_{y} \\
v_{z}=\frac{\mu_{o}^{2} B}{\left(1+\mu_{o}^{2} B^{2}\right)} E_{x}+\frac{\mu_{o}}{\left(1+\mu_{o}^{2} B^{2}\right)} E_{z}
\end{gathered}
$$

In matrix writing, we get:

$$
\left(\begin{array}{l}
v_{x} \\
v_{y} \\
v_{z}
\end{array}\right)=\left(\begin{array}{ccc}
\frac{\mu_{o}}{\left(1+\mu_{o}^{2} B^{2}\right)} & 0 & -\frac{\mu_{o}^{2} B}{\left(1+\mu_{o}^{2} B^{2}\right)} \\
0 & \mu_{o} & 0 \\
\frac{\mu_{o}^{2} B}{\left(1+\mu_{o}^{2} B^{2}\right)} & 0 & \frac{\mu_{o}}{\left(1+\mu_{o}^{2} B^{2}\right)}
\end{array}\right)\left(\begin{array}{c}
E_{x} \\
E_{y} \\
E_{z}
\end{array}\right)
$$

Furthermore the current density is the phoconductivity tensor times the electric field as given by Equation (9):

$$
\boldsymbol{J}=(\sigma) \boldsymbol{E}
$$

where:

$$
(\sigma)=\frac{q \delta_{n} \mu_{o}}{\left(1+\mu_{o}^{2} B^{2}\right)}\left(\begin{array}{ccc}
1 & 0 & -\mu_{o} B \\
0 & \left(1+\mu_{o}^{2} B^{2}\right) & 0 \\
\mu_{o} B & 0 & 1
\end{array}\right)
$$

The parameter $\mu_{o}=\frac{q \tau}{m}$ is the initial mobility of the electrons in the base without magnetic field; $\delta_{n}$ is the excess minority carriers' density.

To simplify our study, a one dimension along the $x$ axis is considered. The expression of the photoconductivity then becomes:

$$
\sigma_{p h}=\frac{q \mu_{o} \delta_{n}}{\left(1+\mu_{o}^{2} B^{2}\right)}
$$

In Equation (11), the excess minority carriers' density is necessarily required. Thus, in steady-state, the evolution of the minority carriers' density is governed 
by the continuity equation:

$$
D_{n} \frac{\partial^{2} \delta_{n}}{\partial x^{2}}-\frac{\delta_{n}}{\tau}=-G
$$

where $D_{n}$ is the minority carriers' diffusion and its expression can be written as:

$$
D_{n}=\frac{D_{o}}{1+\mu_{o}^{2} B^{2}}
$$

where $D_{o}$ is the minority carriers' diffusion coefficient without magnetic field; $G$ is the minority carriers' generation rate [23] at the position $X$, whose expression is:

$$
G=\eta \sum_{i=1}^{3} a_{i} \mathrm{e}^{-b_{i} x}
$$

with $\eta$ the solar number, $a_{i}$ and $b_{i}$ are coefficients deduced from modelling of the generation rate considered over all solar radiation spectrum under AM 1.5.

The solution of Equation (12) is given by:

$$
\delta_{n}=A \mathrm{e}^{-\frac{x}{L_{n}}}+C \mathrm{e}^{\frac{x}{L_{n}}}-\sum_{i=1}^{3} \frac{a_{i} L_{n}^{2} \mathrm{e}^{-b_{i} x}}{D_{n}\left(b_{i}^{2} L_{n}^{2}-1\right)}
$$

with:

$$
L_{n}=\sqrt{D_{n} \tau} \text { et }\left(b_{i}^{2} L_{n}^{2}-1\right) \neq 0
$$

where $L_{n}$ is the minority carriers diffusion length in the base.

The coefficients $A$ and $C$ are determinated by the following boundary conditions [24] [25]:

- at the junction $(x=0)$

$$
\left.D_{n} \cdot \frac{\partial \delta_{n}}{\partial x}\right|_{x=0}=\left.S f \cdot \delta_{n}\right|_{x=0}
$$

- at the rear side $(x=H)$

$$
\left.D_{n} \cdot \frac{\partial \delta_{n}}{\partial x}\right|_{x=H}=-\left.S b \cdot \delta_{n}\right|_{x=H}
$$

where $S f$ and $S b$ are respectively minority carrier junction and back surface recombination velocities. The recombination velocity $S f$ is the sum of the junction recombination velocity due to the external load that defines the solar cell operating point and the intrinsic junction recombination velocity that is an effective recombination velocity at the emitter-base interface.

\section{Results and Discussion}

\subsection{Profile of the Photoconductivity According to the Base Depth}

Figure 2 presents the profile of the photoconductivity according to the base depth for different values of the applied magnetic field:

We note that, for each curve data, the photoconductivity decreases with the increase of the base depth. In the vicinity of the junction, with a defined operating 


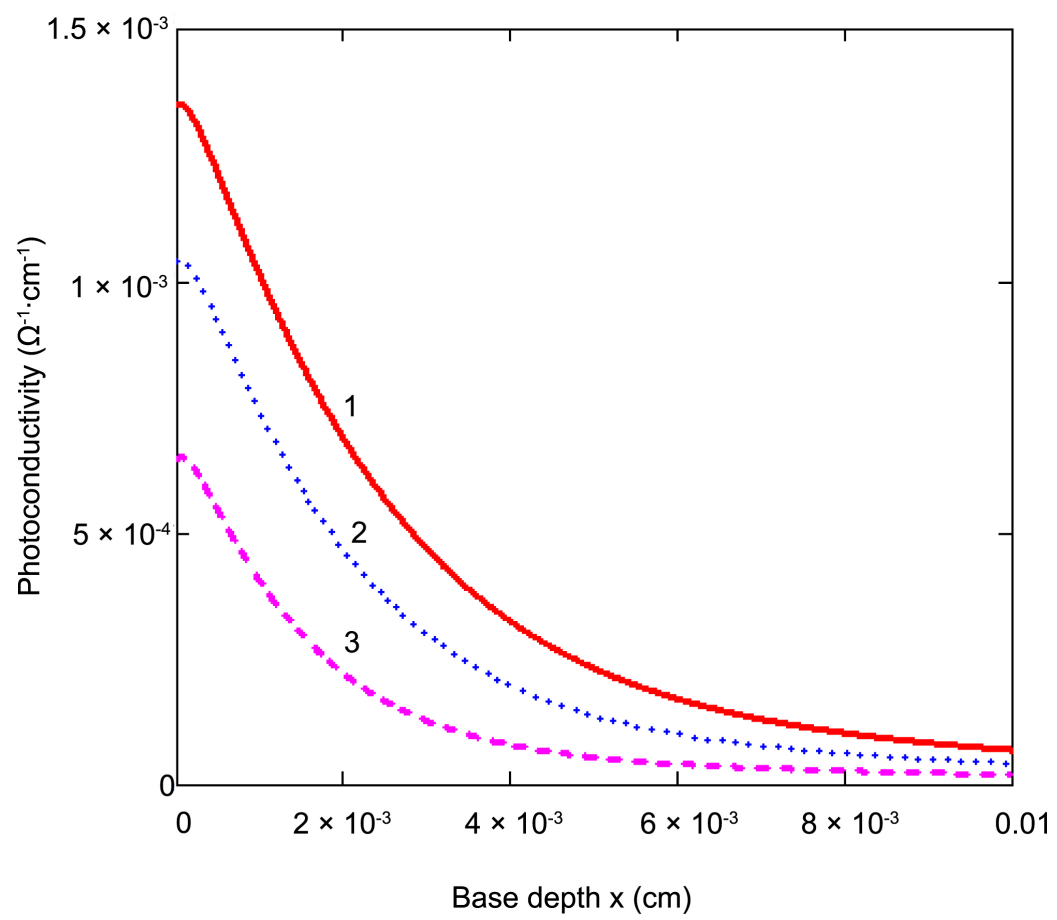

Figure 2. Photoconductivity versus base depth for different magnetic field values 1) $B=0 \mathrm{~T}$; 2) $B=5 \times 10^{-4} \mathrm{~T}$; 3) $B=10^{-3} \mathrm{~T}$.

point around $\mathrm{Sf}=2 \times 10^{2} \mathrm{~cm} \cdot \mathrm{s}^{-1}$, the photoconductivity is maximum for each curves since the density of the minority carriers is also maximum. But, when the base depth increases, the absorption of the incident photons is weak; accordingly the low minority carriers photogeneration and their recombination in volume, leads to a decrease of the photoconductivity as predicted by the Beer-Lambert law. With an applied magnetic field, the photoconductivity decreases because of the magneto-photoconductivity phenomenon and the deflected minority carriers towards the surface. For an optimal use of such solar cell in optoelectronics, laser or detector, it is necessary to choose a base thickness less or equal to $2 \times$ $10^{-3} \mathrm{~cm}(\approx 20 \mu \mathrm{m})$ for a better incident photons absorption and then a decrease of recombination in volume. To highlight the magneto-photoconductivity, we present the behavior of the photoconductivity with the applied magnetic field.

\subsection{Profile of the Photoconductivity According to the Logarithm of Magnetic Field}

In Figure 3, the photoconductivity is represented versus the logarithm of the applied magnetic field for different base depth:

For each curve, the behavior of the photoconductivity is the same. In the interval of the magnetic field $\left[0 \mathrm{~T} ; 10^{-4} \mathrm{~T}\right]$, the photoconductivity is constant and stays at its maximum value for a given curve. Here, the effect of the magnetic field is weak on the photo generated minority carriers. The magnetic force that acts on minority carriers, is so weak that there is no significant deflected carriers that could be recombined in surface area or in the bulk of the base. In contrast in 


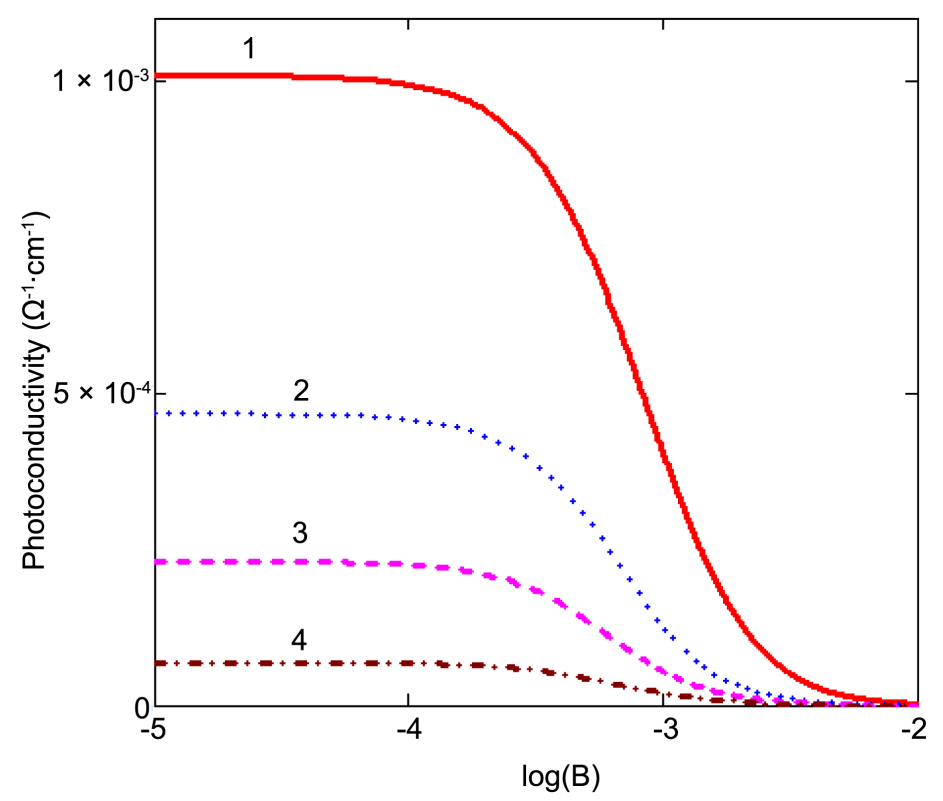

Figure 3. Photoconductivity versus logarithm of magnetic field for different depths: 1) $x=10^{-3} \mathrm{~cm}$; 2) $x=3 \times 10^{-3} \mathrm{~cm}$; 3) $x=5 \times 10^{-3} \mathrm{~cm}$; 4) $x=10^{-2} \mathrm{~cm}$.

the interval $\left[10^{-4} \mathrm{~T} ; 10^{-2} \mathrm{~T}\right]$, the photoconductivity decreases markedly because of the strong magnetic force which acts on the minority carriers trajectories that are more and more incurvated. The increase of the magnetic field while decreasing photoconductivity, induced change in the solar cell properties by creating degenerated levels states.

We now show the behavior of the photoconductivity with the operating point of the solar cell.

\subsection{Profile of the Photoconductivity According to the Junction Recombination Velocity}

We present in Figure 4, the behavior of the photoconductivity according to the junction recombination velocity for different applied magnetic field values.

We note that, for each curve in Figure 4, the photoconductivity decreases with the increase of the junction recombination velocity. We observe, for a given magnetic field, three regions: - the first corresponds to the solar cell operating point in open circuit $\left[0 \mathrm{~cm} \cdot \mathrm{s}^{-1} ; 2 \times 10^{2} \mathrm{~cm} \cdot \mathrm{s}^{-1}\right]$, where the photoconductivity is quite constant. The minority carriers have not enough kinetic energy to cross the junction and this leads to their storage in the vicinity of the junction: - the second corresponds to the solar cell variable operating point $\left[2 \times 10^{2} \mathrm{~cm} \cdot \mathrm{s}^{-1} ; 5 \times\right.$ $10^{5} \mathrm{~cm} \cdot \mathrm{s}^{-1}$, where the photoconductivity decreases with respect to the junction recombination velocity. The photogenerated minority carriers have gradually some kinetic energy that allows them to cross the junction while the load decreases. This situation leads to the decrease of the minority carriers stored in the vicinity of the junction; - the third corresponds to the solar cell operating point 


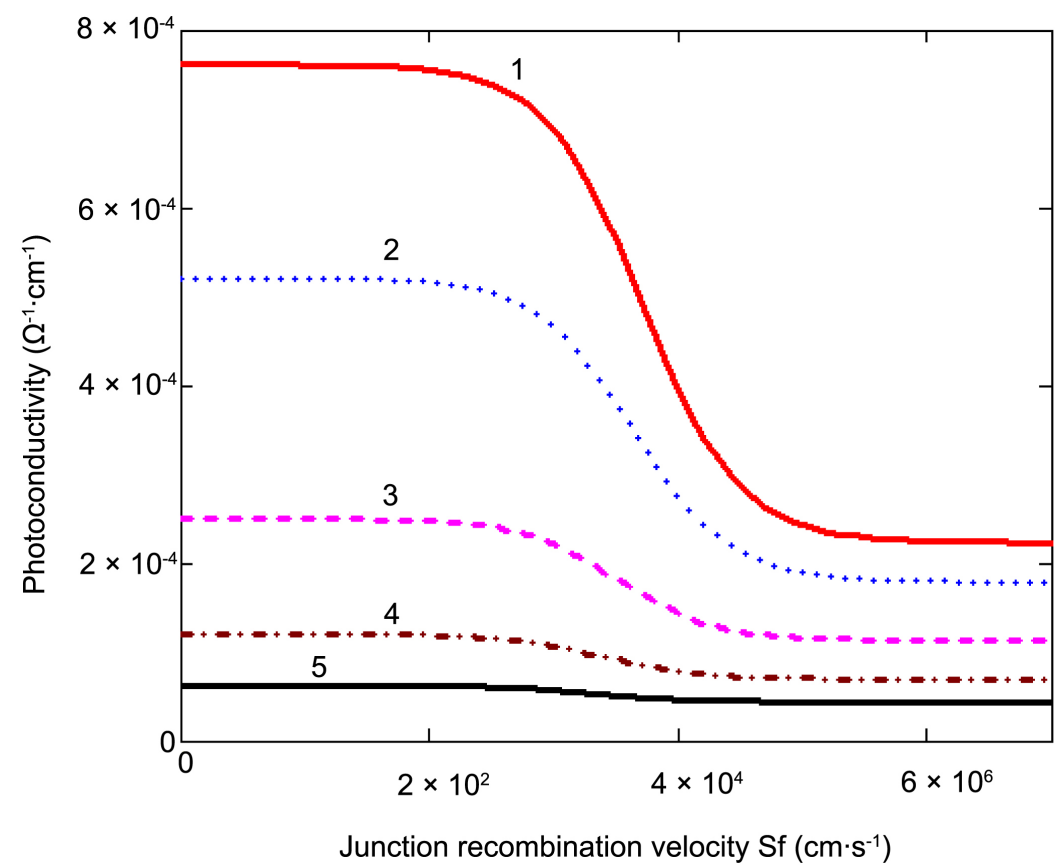

Figure 4. Photoconductivity versus junction recombination velocity for different magnetic field values. 1) $B=0 \mathrm{~T}$; 2) $B=5 \times 10^{-4} \mathrm{~T}$; 3) $B=10^{-3} \mathrm{~T}$; 4) $B=1.5 \times 10^{-3}$ T; 5) $B=2 \times 10^{-3} \mathrm{~T}$.

in short circuit situation $\left[5 \times 10^{5} \mathrm{~cm} \cdot \mathrm{s}^{-1} ; 7 \times 10^{7} \mathrm{~cm} \cdot \mathrm{s}^{-1}\right]$. The photo created minority carriers have very high kinetic energy to cross the junction. There are no more significant stored minority carriers and the photoconductivity remains constant but is lower than those obtained in open circuit situation. For all these above cases, an applied magnetic field causes a decrease in the magnitude of the photoconductivity. Therefore, for an application in optoelectronics, laser or detector, we have to select the solar cell operating point before its short-circuit situation. In order to determine the solar cell electrical parameters, in the interval $\left[2 \times 10^{2} \mathrm{~cm} \cdot \mathrm{s}^{-1} ; 5 \times 10^{5} \mathrm{~cm} \cdot \mathrm{s}^{-1}\right]$, we can apply the linear model of this part of the photoconductivity curve since there is a straight line observed. Thus, we obtain a relationship between the photoconductivity and the junction recombination velocity as:

$$
\sigma_{p h}=-C \cdot S f+\sigma_{p h o}
$$

where $\sigma_{\text {pho }}$ is the photoconductivity in open circuit situation; $-C$ is the slope of the linear part and $C$ being the electrical capacitance.

In Table 1, we give a few values of $C$ and $\sigma_{p h o}$ for three magnetic field values.

We note a decrease of both electrical capacitance and photoconductivity in open circuit situation with the increase of the applied magnetic field. The decrease of the diffusion capacitance is linked to the space charge region (in the vicinity of the junction) increase since there is no significant stored minority carriers and the occurring of the magnetoresistance effect on the solar cell. 
Table 1. Values of $C$ and $\sigma_{\text {pho }}$ for different magnetic fields.

\begin{tabular}{cccccc}
\hline$B(\mathrm{~T})$ & 0 & $5 \times 10^{-4}$ & $10^{-3}$ & $1.5 \times 10^{-3}$ & $2 \times 10^{-3}$ \\
\hline$\sigma_{\text {pho }}\left(10^{-4} \Omega^{-1} \cdot \mathrm{cm}^{-1}\right)$ & 7.62 & 5.21 & 2.50 & 1.2 & 0.6 \\
$C\left(\mathrm{pF} \mathrm{cm}^{-2}\right)$ & 894 & 555 & 210 & 73 & 25 \\
\hline
\end{tabular}

\section{Conclusion}

A steady-state of the photoconductivity of a bifacial silicon solar cell has been presented. The Drüde model and the continuity equation permitted to determine the photoconductivity according to the base depth, the applied magnetic field and the junction recombination velocity. The effects of the depth, the magnetic field and the junction recombination velocity, have enabled us to determine an optimum base depth and to be in open circuit situation for having more photoconductivity. By using a linear model of the photoconductivity, we have determined an electrical capacitance, which decreases with the applied magnetic field. The method of the steady photoconductivity has been used to characterize a thin film silicon solar cell capacitance. For the future works, few parameters, such series and shunt resistances, quantum efficiency, spectral response will be considered in $1 \mathrm{D}$ or $3 \mathrm{D}$, taking into account of the grain boundaries recombination. This method will be utilized to heterojunctions solar cells in transient regime, frequency modulation with or without magnetic field effect.

\section{References}

[1] Bube, R.H. (1960) Photoconductivity of Solids. Wiley, New York.

[2] Bube, R.H. (1974) Electronic Properties of Crystalline Solids. Academic Press, New York.

[3] Joshi, N.V. (1990) Photoconductivity: Art, Science and Technology. Marcel Dekker, New York.

[4] Orton, J. (2004) The Story of Semiconductors. Oxford University Press, Oxford.

[5] Adriaenssens, G.J., Baranovskii, S.D., Fuhs, W., Jansen, J. and Öktü, Ö. (1995) Physical Review B, 51, 9661-9667. https://doi.org/10.1103/PhysRevB.51.9661

[6] Belgacem, H. and Merazga, A. (2008) Solid-State Electronics, 52, 73-77. https://doi.org/10.1016/j.sse.2007.07.023

[7] Sinton, R.A. and Cuevas, A. (1996) Applied Physics Letters, 69, 2510-2512. https://doi.org/10.1063/1.117723

[8] Feng, Q., Xiang, J.Z., Kong, J.C., Yu, L.J., Kong, L.D., Wang, G.H., Li, X.J., Yang, L.L., Li, C. and Ji, R.B. (2011) Journal of Semiconductors, 32, 0330041-0330045.

[9] Qamhieh, N. and Adriaenssens, G.J. (2001) Journal of Non-Crystalline Solids, 292, 80-87. https://doi.org/10.1016/S0022-3093(01)00855-9

[10] Pal, R.K., Ji, K., Agnihotri, A.K., Singh, C.P., Yadav, S. and Kumar, A. (2009) Chalcogenide Letters, 6, 29-34.

[11] Reis, F.T. and Chambouleyron, I. (2002) Journal of Non-Crystalline Solids, 299-303, 179-184. https://doi.org/10.1016/S0022-3093(01)01159-0

[12] Sharmin, M., Choudhury, S., Akhtar, N. and Begum, T. (2012) Journal of Bangladesh Academy of Sciences, 36, 97-107. https://doi.org/10.3329/jbas.v36i1.10926 
[13] Schmidt, J.A., Longeaud, C. and Kleider, J.P. (2005) Thin Solid Films, 493, 319-324. https://doi.org/10.1016/j.tsf.2005.08.060

[14] Omar, M.S. and Abbas, T.A. (2010) Iranian Journal of Physics Research, 9, 99-102.

[15] Heisel, W., Bohm, W. and Prettl, W. (1981) International Journal of Infrared and Millimeter Waves, 2, 829-837. https://doi.org/10.1007/BF01007279

[16] Meier, D.L., Hwang, J.-M. and Campbell, R.B. (1998) IEEE Transactions on Electron Devices, ED-35, 70-78.

[17] Hüber, A., Aberle, A.G. and Hezel, R. (2001) 20\% Efficient Bifacial Silicon Solar Cells. Munich 14th European Photovoltaic Solar Energy Conference, 1796-1798.

[18] Madougou, S., Made, F., Boukary, M.S. and Sissoko, G. (2007) Advanced Materials Research, 18-19, 303-312.

https://doi.org/10.4028/www.scientific.net/AMR.18-19.303

[19] Thiam, Nd., Diao, A., Ndiaye, M., Dieng, A., Thiam, A., Sarr, M., Maiga, A.S. and Sissoko, G. (2012) Research Journal of Applied Sciences, Engineering and Technology, 4, 4646-4655.

[20] Madougou, S., Made, F., Boukary, M.S. and Sissoko, G. (2007) Advanced Materials Research, 18-19, 313-324.

[21] Charles Kittel (1972) Introduction à la Physique de l'état solide. Dunod, Université, 284-286.

[22] Diao, A. and Sissoko, G. (2017) Journal of Material Sciences \& Engineering, 6, 381. https://doi.org/10.4172/2169-0022.1000381

[23] Mohammad, S.N. (1987) Journal of Applied Physics, 61, 767-772. https://doi.org/10.1063/1.338230

[24] Sissoko, G., Nanéma, E., Corréa, A., Biteye, P.M., Adj, M. and N’Diaye, A.L. (1998) Silicon Solar Cell Recombination Parameters Determination using the Illuminated I-V Characteristic. World Renewable Energy Congress, 1847-1851.

[25] Sissoko, G., Sivoththanam, S., Rodot, M. and Mialhe, P. (1992) Constant Illumination-Induced Open Circuit Voltage Decay (CIOCVD) Method, as Applied to High Efficiency Si Solar Cells for Bulk and Back Surface Characterization. 11 th European Photovoltaic Solar Energy Conference and Exhibition, Montreux, 352-354. 